\title{
Social-Media-Strategie - Ressourcenplanung, Budget und die richtige Kommunikation
}

\section{Daniela Vey}

2009 führte Facebook Fanpages für Marken, Unternehmen und Kommunen ein. Damit wurde der Grundstein für die professionelle Social-Media-Arbeit von heute gelegt. Die Entwicklungen nahmen über die Jahre entscheidend Einfluss auf die Gesellschaft und Politik und veränderten den Austausch von Informationen, Erfahrungen und Sichtweisen.

Anforderungen an kommunale und öffentliche Auftritte wachsen und verändern sich. Die Systeme der einzelnen sozialen Netzwerke verändern sich schneller und werden zunehmend komplexer. Nicht immer ist es einfach, in diesem Dschungel der Möglichkeiten den Überblick zu behalten.

In der Arbeit mit Kommunen kommen dabei drei Themen immer wieder auf: Es mangelt an Zeit, eigenem Budget und strategischem Fachwissen. Dem entgegen steht die Erkenntnis, dass man dort vertreten sein muss, wo sich die Bürger bewegen. Man möchte an den Gesprächen teilnehmen und auch die Menschen erreichen, die weder Tageszeitung noch Amtsblatt lesen.

In der Regel wird jemand zusätzlich zu seinem eigentlichen Job mit dem Projekt ,Social Media' betraut, oft unabhängig von Interesse oder Vorkenntnissen. Da die sozialen Medien eine besondere Art der Kommunikation erfordern, beißt sich hier häufig die Katze in den Schwanz. Fühlt sich jemand unsicher oder gar unwohl in diesen Kommunikationskanälen, lässt sich das zwischen den Zeilen erkennen. Dies kann wiederum dazu führen, dass die Interaktionen schwach oder

D. Vey $(\bowtie)$

Infodesignerin.de, Stuttgart, Deutschland

E-Mail:dv@infodesignerin.de 
im schlimmsten Fall sogar negativ ausfallen. Dies wiederum bestärkt die Kritiker im Haus oder in der Kommune, die das Ganze ohnehin für eine schlechte Idee gehalten haben. Es herrschen diffuse Ängste vor zusätzlichem Arbeitsaufwand, Shitstorms und der damit einhergehenden Verantwortung.

Gleichzeitig ist wichtig, dass die Chefetage wirklich hinter dem Social-MediaAuftritt steht, die Verantwortung mitträgt und über den realistischen Arbeits- und Kostenaufwand informiert ist. Diese Rückendeckung erleichtert die Arbeit erfahrungsgemäß deutlich.

\section{Zeit}

\section{Personalplanung}

Zeitmangel stellt einen kritischen Faktor in Verwaltungen dar. Viele Verantwortliche übernehmen die Pflege der Social-Media-Kanäle als zusätzliche Aufgabe zu ihrem eigentlichen Job.

Auch der Führungsebene sollte klar sein, dass hier Arbeitszeit aufgewendet wird, die man berücksichtigen und einplanen muss. Je nach Anzahl der Kanäle, Interaktionsraten und Abstimmungsprozessen im Haus sind zwei bis acht Stunden pro Woche notwendig.

Empfehlenswert ist die Organisation im Team, insbesondere wenn keine eigene Stelle für Social-Media-Arbeit vorgesehen ist. Das verteilt die anfallenden Aufgaben auf mehrere Schultern und entlastet den Einzelnen. Gemeinsam übernimmt man Verantwortung, ergänzt sich in seinen Kompetenzen und kann sich im Fall von Urlaub, Krankheit oder zu hoher Arbeitslast gegenseitig vertreten. Hinzu kommt, dass strategische Entscheidungen leichter zu treffen sind, wenn man sich dazu im Team besprechen kann.

Es erleichtert die Zusammenarbeit, wenn die ausgewählten Personen grundsätzlich am Thema Social Media interessiert und von sich aus dort aktiv sind. Schulungen zu den jeweiligen Netzwerken bauen das bereits vorhandene Wissen aus. Inhaltlich sollte es dabei um die technische Bedienung, Chancen und Risiken sowie die ausgearbeitete Kommunikationsstrategie der Kommune gehen. Je besser der Grundstein hier gelegt wird, umso effektiver und unabhängiger (von Dienstleistern) kann das Team arbeiten. 


\section{$2 \quad$ Teamaufstellung}

\subsection{Das Kernteam}

besteht aus zwei bis drei kommunikationserfahrenen Personen, die beispielsweise für Pressestelle, Online-Kommunikation oder Stadtmarketing verantwortlich sind. Sie sollten sich aus beruflichem Interesse in den jeweiligen Netzwerken aktiv bewegen, um ein gutes Gefühl für Sprache, Bildauswahl und aktuelle Themen zu haben. Vorkenntnisse in den jeweiligen Kanälen sind von Vorteil, lassen sich aber bei Bedarf durch entsprechende Schulungen ergänzen.

Typische Aufgaben des Kernteams sind beispielsweise

- Festlegen der Gesamtstrategie

- Entscheidungen über Auswahl der Kanäle

- Erstellen und Freigeben von Beiträgen

- Auswerten der Statistiken

- Beantworten von Fragen und Nachrichten

- Moderieren der Kommentare

- Beauftragung von Dienstleistern (wenn notwendig)

\subsection{Das Redaktionsteam}

besteht aus vier bis sechs Personen aus unterschiedlichen Bereichen - sinnvollerweise mit bürgernahen Themen wie Bürgerbüro, Bauamt, Fundbüro, Kinder- und Jugendarbeit, Kultur oder Stadtbibliothek. Die Ausgewählten sollten Begeisterung und Affinität zu den jeweiligen Netzwerken mitbringen, da dies die Zusammenarbeit ungemein erleichtert. Mit ein wenig Übung und Abstimmung können die Mitglieder des Redaktionsteams später selbstständig Beiträge veröffentlichen und Anfragen bearbeiten. So entlasten sie das Kernteam.

Typische Aufgaben sind hier

- Vorbereiten und Erstellen von Beiträgen

- Beantworten von Fragen und Nachrichten

- Moderieren der Kommentare 


\subsection{Das Content-Team}

ist breit aufgestellt und ergänzt die noch fehlenden Themenbereiche. Es ist wichtig, dass es zumindest in jedem Amt bzw. jeder Abteilung einen Ansprechpartner gibt, der auch für die Zulieferung von Themen verantwortlich ist. Derjenige sollte wissen, worauf es bei der Auswahl der Inhalte für das jeweilige Netzwerk ankommt, um so die wichtigsten Informationen an Redaktions- und Kernteam zu liefern. Diese übernehmen das Verfassen und Veröffentlichen des Beitrags. Bei Rückfragen hat man so einen festen Ansprechpartner im Fachbereich.

Typische Aufgaben des Content-Teams sind

- Auswahl von sinnvollen Themen

- Vorbereitung für das Kernteam

- Beantworten von Rückfragen

- Moderieren der Kommentare

Gut geplant ist halb gewonnen - Der Redaktionsplan

$\mathrm{Zu}$ einem gut organisierten Team gehört eine durchdachte Redaktionsplanung. Zielsetzung sollte es sein, in jedem der ausgewählten Netzwerke regelmäßig Beiträge zu veröffentlichen. Sollte das nicht möglich sein, benötigt man entweder mehr Ressourcen oder sollte die Anzahl der vorhandenen Social-Media-Kanäle hinterfragen.

\section{Redaktionsplanung}

\section{Weiche Themen}

Ideen für unterhaltsame und wiederkehrende Themen sollte man an einem festen Ort sammeln, z. B. im Rahmen des Redaktionsplanes, auch weil sich nicht alle davon direkt umsetzen lassen. Durch das Festschreiben in einem Redaktionsplan kann man bei Bedarf immer wieder auf diese zurückgreifen und sie als Inspiration nutzen, um ,Löcher' in der Planung zu vermeiden.

Unterhaltsam und immer wieder gerne gesehen sind historische Bilder, Zitate, Anekdoten der Stadtgeschichte, Vorstellung von bekannten Persönlichkeiten oder Serien wie ,Unnützes Wissen'. Aber auch die Vorstellung von Mitarbeitern, ihren Aufgaben bzw. Stellen können spannend sein - gerade, wenn es darum geht Nachwuchs- und Fachkräfte zu gewinnen. Für die einzelnen Themen sind unterschiedliche Frequenzen denkbar: wöchentlich, monatlich oder als kleine Serie z. B. in den Sommerferien (beispielsweise der Nürtinger Schlemmer Sommer 2018). 


\section{Langfristig: Jahresplanung}

Hierzu gehören Feiertage, Jubiläen, Stadtfeste, Messen und wichtige Termine für die Bürger, z. B. die fristgerechte Erinnerung an die Verlängerung von Reisepässen vor den Sommerferien. Auch ,internationale Tage' können wunderbare Aufhänger für bestimmte Themen sein, z. B. Tag des Denkmals, Tag des Buches, Tag der Freundschaft oder Tag der Jogginghose. Es sollte stets überlegt werden, was zum eigenen Auftritt passt, aber gerade hier bietet sich auch die Chance kreativ und gerne auch humorvoll zu werden.

\section{Mittelfristig: Drei-Monats-Planung}

Anstehende Events werden nun enger begleitet, das Programm detaillierter vorgestellt und u. U. auch mit Werbekampagnen die Reichweite erhöht. Dabei ist Wiederholung wichtig: Menschen brauchen im Durchschnitt sieben bis 12 Kontaktpunkte, bevor sie eine Entscheidung treffen. Auf ausgeschriebene Stellen sollte vor Ablauf der Bewerbungsfrist regelmäßig hingewiesen und das Berufsbild vorgestellt werden. Auch Servicehinweise an die Bürger wie Fristen für die Verlängerung der Reisepässe vor den Sommerferien machen an dieser Stelle Sinn.

\section{Kurzfristig: Wochen- bzw. Monatsplanung}

Beiträge werden ganz konkret geplant: Tag, Uhrzeit, Thema, Ansprechpartner. Es sollte trotzdem immer ein wenig Raum bleiben, um auf aktuelle Ereignisse reagieren zu können. Deshalb ist es wichtig zu jedem Beitrag im Redaktionsplan festzuhalten, ob der gewählte Zeitpunkt flexibel ist und verschoben werden kann oder nicht.

Bei der Auswahl der Themen sollte man das aktuelle Zeitkontingent des Teams berücksichtigen. Es gibt Themen, die ein höheres Maß an Diskussion auslösen und moderiert werden müssen, z. B. Verkehr oder Flüchtlinge. Dies fließt als Faktor in die Planung mit ein, da der Ansprechpartner greifbar sein muss und zeitnahes Antworten ermöglichen sollte. Solche Beiträge platziert man besser zu Beginn der Woche als am Freitag kurz vor Feierabend.

Falls aufgrund äußerer Umstände ein ungünstiger Zeitpunkt gewählt werden muss, gilt es vorab folgende Fragen zu klären:

- Wer sind weitere Ansprechpartner im Haus bei Rückfragen?

- Wie geht man mit zusätzlichen Arbeitszeiten nach Feierabend oder am Wochenende um, wenn es die Umstände erfordern?

- Wer kann das Team zusätzlich unterstützen?

- Kann man auf der Website ausführliche Informationen bereitstellen, auf die die Social-Media-Verantwortlichen bei Bedarf verweisen können?

- Kann man Antworten auf die häufigsten Fragen vorab vorbereiten? 
Der Redaktionsplan hilft, einen guten Überblick über die eigenen Aktivitäten zu wahren. Regelmäßigkeit und eine gleichmäßige Verteilung spielen dabei ebenso eine Rolle, wie die richtige Mischung der Themen und die passende Frequenz der Wiederholungen.

\section{Redaktionssitzung}

Spätestens alle vier Wochen sollte es eine gemeinsame, etwa einstündige Redaktionssitzung geben. Hierbei sollten alle Beteiligten an einem Tisch sitzen: Kernteam, Redaktionsteam und möglichst viele Personen aus dem Content-Team. Die drei notwendigen Punkte der Tagesordnung sind:

\section{Rückblick und Analyse}

Es werden die erfolgreichsten und die schwächsten Beiträge der letzten vier Wochen besprochen. Statistiken dazu stellt jedes Netzwerk bereit. Die stetige Analyse hilft, die eigenen Beiträge weiter zu optimieren. Berücksichtigen kann man z. B. Zeitpunkt der Veröffentlichung, Medienauswahl und Tonalität des Textes.

2. Die nächsten drei Monate

Für wichtige Themen und Veranstaltungen sollte man frühzeitig beginnen, darauf hinzuweisen, Beiträge zu veröffentlichen und bei Bedarf für eine Werbekampagne das entsprechende Budget bereitzustellen.

\section{Die nächsten vier Wochen}

Die Beiträge der nächsten vier Wochen werden ganz konkret geplant: Zeitpunkt, Inhalt, benötigte Medien wie Bilder, Videos oder Links zu weiterführenden Informationen sowie klare Verantwortlichkeiten werden festgelegt. Besonders der letzte Punkt ist wichtig: Die Aufgaben werden immer an eine Person vergeben und nicht an ein Amt oder eine Abteilung. Außerdem ist zu klären, ob weitere Dinge vorzubereiten sind, wie z. B. Informationen auf der Website, die Bereitstellung von Ausrüstung oder eine Terminvereinbarung mit dem gewünschten Ansprechpartner.

\section{$3 \quad$ Budget}

Profile in verschiedenen Netzwerken zu erstellen ist kostenlos. Das darüber hinaus aber langfristig Investitionen notwendig sind, ist häufig nicht klar. Aus der Spielwiese mit Gratis-Reichweite ist ein professionelles und komplexes Arbeitsfeld geworden. Damit einher geht eine entsprechend notwendige Haushaltsplanung. 
Personalkosten Monatlich beansprucht die Pflege von Social-Media-Kanälen zwischen acht und $24 \mathrm{~h}$ - abhängig von Anzahl der Kanäle, Interaktionsraten und Abstimmungsprozessen im Haus. Das sollte in Form von Personalkosten in die jährliche Haushaltsplanung des jeweiligen Amtes mit aufgenommen werden, wie das Beispiel der Stadt Ludwigsburg zeigt: 93.000 Einwohner und 22.400 Fans auf Facebook hat die Stadt auf ihrem Kanal. Der Hauptverantwortliche Clemens Flach sitzt in der Pressestelle und darf 15-30 \% seiner Arbeitszeit für den Facebook-Auftritt aufwenden. An Personalkosten plant die Stadt jährlich 16.000 EUR ein. Zusätzlich gibt es Budget für Werbung und Workshops. Es gibt noch weitere Social-Media-Auftritte z. B. für Tourismus, Stadtmuseum oder Stadtbibliothek, die aber eigenständig von deren Teams betreut werden.

Aus- und Weiterbildung Im Social-Media-Bereich ist das Tempo der Neuentwicklungen sehr hoch. Die Netzwerke und ihre Möglichkeiten entwickeln sich permanent weiter. Das hat sowohl Auswirkungen auf deren technische Bedienung als auch auf die Strategie. Es ist daher von Bedeutung, ständig am Ball zu bleiben und den eigenen Kanal permanent weiterzuentwickeln. Der Erfahrungsaustausch mit anderen Verantwortlichen kann hier sehr hilfreich sein.

Es gibt eine ganze Reihe von guten Blogs und Newslettern. Zusätzlich sollte Budget für fachliche Fortbildungen, Inhouse-Workshops mit Experten oder Fachkonferenzen eingeplant werden. Das Angebot dahin gehend ist mittlerweile vielfältig. $\mathrm{Zu}$ den günstigeren Optionen gehören BarCamps und Social-Media-Nights, die es in vielen größeren Städten gibt. Bei Konferenzen ist man dagegen schnell im drei- bis vierstelligen Bereich. Diese sind dafür in der Regel sehr effektiv und vermitteln viel Wissen und Inspiration in kurzer Zeit.

Die Investition, eigenes Wissen auf- und auszubauen lohnt sich, da man sich langfristig unabhängiger von Agenturen und anderen Dienstleistern macht. Punktuell kann es jedoch dennoch sinnvoll sein, Spezialwissen von außen einzukaufen oder bestimmte Aspekte wie z. B. die Schaltung von Werbekampagnen auszulagern.

\section{Ausrüstung}

Eine gute Ausrüstung wirkt sich positiv auf die Effizienz der Arbeitsprozesse aus. So lassen sich mit den richtigen Werkzeugen viele Dinge beschleunigen und vereinfachen. 
Ein Rechner mit aktueller Browserversion Viele Fachanwendungen in Verwaltungen sind auf ältere Browserversionen optimiert. Dementsprechend werden diese von der IT ausgewählt und installiert. Netzwerke wie Facebook oder Twitter funktionieren in aktuellen Browser-Versionen allerdings deutlich besser und schneller. Daher ist sinnvoll, in solchen Fällen einen zusätzlichen Browser für die Social-Media-Arbeit installieren zu lassen.

Ein Smartphone oder Tablet mit mobilen Daten Ein mobiles Dienstgerät bietet viele Vorteile. Die heutigen Tablets und Smartphones sind häufig mit guten Kameras ausgestattet, deren Funktionen sich über Apps noch zusätzlich optimieren und erweitern lassen. So hat man Kamera und Bild- bzw. Videobearbeitung in einem Gerät vereint. Außerdem können so Beiträge zeitnah oder live veröffentlicht werden, z. B. Eindrücke vom Stadtfest oder eine Fragestunde mit dem Bürgermeister.

Hinzu kommt, dass Instagram derzeit viele seiner Funktionen ausschließlich über die App zur Verfügung stellt. Man benötigt zusätzliche, z. T. kostenpflichtige Tools, um auch über einen Rechner Inhalte einstellen zu können.

Redaktionstools Die Redaktionsplanung lässt sich mit einfachen Mitteln wie Excel oder dem kostenlosen Tool ,Trello" realisieren. Durch diese ergeben sich allerdings keine Workflows oder Freigabe-Prozesse. Dafür gibt es kostenpflichtige Tools, die die Pflege mehrerer Profile unterstützen und außerdem zusätzliche Auswertungen und Statistiken bieten. Das kann gerade in einem dezentral organisierten Team die Arbeitsprozesse erleichtern.

Die Ausrüstung lässt sich je nach Budget beliebig erweitern: Von professionellen Kameras und Mikrofonen über Bildbearbeitungs- und Videoschnitt-Software bis hin zu Monitoring- und Management-Tools. Die oben genannten drei sollten aber als Basis-Ausrüstung jedem Social-Media-Team zur Verfügung stehen.

Werbung Die Konkurrenz im Bereich Social Media wächst stetig. Neben Unternehmen und Marken nehmen auch Influencer einen immer größeren Raum ein. Nach einigen Skandalen räumt zumindest Facebook im Newsfeed vertrauenswürdigen lokalen Seiten und Nachrichten eine höhere Priorität ein. Von dieser Entwicklung profitieren Städte und die öffentliche Verwaltung in Hinblick auf ihre Reichweite.

Dennoch wird es schwieriger, sich organisch gegen die Konkurrenz zu behaupten. Daher sollte auch ein Werbebudget für wichtige Themen oder Veranstaltungen im Haushalt vorgesehen werden. Je nach Kompetenzen und Möglichkeiten im Haus muss dafür unter Umständen eine Agentur oder Beratung beauftragt werden. 


\section{$4 \quad$ Strategie}

Soziale Netzwerke sind ein Dialog-Medium. Hier geht es nicht wie bei Websites oder Broschüren darum, Informationen $\mathrm{zu}$ veröffentlichen, sondern zu ausgesuchten Themen mit den Bürgern, Fans und Followern ins Gespräch zu kommen.

Erfolgsfaktoren Erfolg definiert sich in sozialen Netzwerken über die Interaktionsrate und die damit verbundene Reichweite. Fanzahlen haben damit wenig zu tun, werden aber leider noch immer viel zu häufig als Indikator für einen ,guten Auftritt' herangezogen. Wer 10.000 Fans hat, aber keine Reaktionen auf seine Beiträge bekommt, wird in sozialen Netzwerken schnell unsichtbar. Je nach Aktivität des Nutzers selbst verschwinden die Beiträge von Seiten, mit denen er nicht interagiert, spätestens nach drei Monaten aus dem Newsfeed und somit aus der Wahrnehmung. Nachhaltiger Erfolg wird durch stetige Interaktion mit der eigenen Community und darüber hinaus bedingt. Dazu sind qualitativ hochwertige und relevante Beiträge in gleichmäßigen Abständen die wichtigste Grundlage.

Der Algorithmus Mittlerweile werden in allen Netzwerken die Beiträge im Newsfeed nicht mehr chronologisch angezeigt, sondern mit Hilfe von Algorithmen personalisiert, priorisiert und umsortiert. Dabei spielen Nutzerverhalten und Interessen eine entscheidende Rolle. Ziel ist es, jedem Nutzer für ihn persönlich interessante und relevante Neuigkeiten zu präsentieren.

Einem durchschnittlichen deutschen Facebook-Nutzer müssten normalerweise etwa 1500 Beiträge täglich angezeigt werden. Das Netzwerk sortiert allerdings - basierend auf dem bisherigen Verhalten - rund $80 \%$ der Inhalte aus und präsentiert dem Nutzer lediglich etwa 300 Meldungen am Tag. Als Seitenbetreiber möchten man natürlich in den verbleibenden $20 \%$ erscheinen und das im Idealfall möglichst weit oben. Aus diesem Grund sind die Interaktionen so wichtig. Je intensiver sich die Nutzer mit den Inhalten der Seite beschäftigen, umso häufiger und früher taucht diese Seite dann auch im Newsfeed auf.

$\mathrm{Zu}$ den gemessenen Interaktionen gehören ,Gefällt mir'-Angaben, Kommentare, Teilen und Klicken auf die Inhalte (Bilder zum Vergrößern oder Links auf Websites, um den zugehörigen Artikel zu lesen). Dabei erhöhen das Teilen und die Kommentierung eines Beitrags die Reichweite stärker als einfache ,Gefällt mir'-Angaben. Die drei wichtigsten Faktoren sind dabei: 
Affinity - Beziehung zwischen Absender und Empfänger Interagiert der Fan häufig mit den Inhalten einer Seite oder schreibt Nachrichten, werden ihm die Beiträge schneller und vor allem regelmäßiger angezeigt. Der Algorithmus erkennt eine enge Beziehung.

Weight - Anzahl der Interaktionen erhöht die Reichweite Wird mit einem bestimmten Beitrag viel und intensiv interagiert, messen ihm die Netzwerke allgemein eine höhere Priorität zu und zeigen ihn organisch mehr Menschen an als Beiträge mit geringer Interaktion. So sieht ihn z. B. auch ein Fan, der länger nicht mit einer Seite interagiert hat.

Decay - Zeitabstand zwischen Veröffentlichung und letztem Login Je kleiner der Abstand zwischen der Veröffentlichung des Beitrags und dem Login der User ist, desto eher wird der Beitrag gesehen und wahrgenommen. Die Statistiken zeigen deutlich, wann die meisten Fans online sind. Typische Zeiten sind vor der Arbeit, in der Mittagspause und nach Feierabend. Der richtige Zeitpunkt spielt eine wichtige Rolle bei der Planung (Roth 2017). So wird beispielsweise ein Beitrag, der um 8 Uhr morgens veröffentlicht wird, nur wenige Fans erreichen, da die meisten Menschen erst gegen $20 \mathrm{Uhr}$ online sind. Wird ein Beitrag allerdings um 19 Uhr veröffentlicht, wird dieser von mehr Nutzer gesehen werden.

Nur sehr gut bewertete Inhalte mit hoher Interaktionsrate werden angezeigt, wenn sie älter sind. Grundsätzlich schafft es kein Beitrag in den Newsfeed, der älter ist als eine Woche. Daraus folgt die Minimal-Anforderung von zwei Beiträgen pro Woche, um die Chance zu wahren, sichtbar zu bleiben. Zusammenfassend sollte man regelmäßig Beiträge zum richtigen Zeitpunkt posten, die relevant für die gewünschte Zielgruppe sind und zu Interaktionen animieren.

Content-Strategie Im Rahmen der Strategie-Erarbeitung gilt es, folgende Punkte klar zu beantworten:

- Warum? - Ziele definieren: Warum bewegt man sich als Stadt in dem jeweiligen Netzwerk? Welche konkreten Ziele werden damit verfolgt?

- Wen? - Zielgruppe definieren: Wen wollen wir erreichen? Je besser man seine Zielgruppe kennt, desto besser kann man diese Menschen mit persönlichen, aktuell relevanten Inhalten ansprechen.

- Wo? - Netzwerke auswählen: Wo hält sich unsere Zielgruppe auf? Welche Netzwerke sind daher relevant und wie kann man die gewünschten Personen dort erreichen? Wie viele Netzwerke kann man vom Workflow her sinnvoll bedienen? 
- Was? - Themen auswählen: Welche Themen passen zu den gesetzten Zielen? Was bewegt die Zielgruppe? Welche Schwierigkeitsstufe und Moderationsaufwand haben die Themen? Welcher Zeitaufwand kann vom Team aktuell bewältigt werden?

- Wie? - Geschichten erzählen: Wie kommen wir ins Gespräch? Wie funktioniert die Kommunikation in diesem Netzwerk, z. B. in Hinblick auf Bildsprache, Hashtags oder Emojis?

- Wann? - Plan machen: Wann erscheinen welche Beiträge? Wie oft und regelmäßig sollen wichtige Themen erscheinen? Wer kümmert sich um welche Medien?

Warum? - Ziele definieren Essenzieller Bestandteil einer Strategie ist das Festlegen von sinnvollen und erreichbaren Zielen. Typische Formulierungen sind:

- Imageverbesserung

- Unterstützung der Öffentlichkeitsarbeit

- Bewerbung von Veranstaltungen

- Jüngere Zielgruppe ansprechen: „(Die Tochter vom) OB hat gesagt, wir brauchen jetzt Instagram.“

Ebenfalls wichtig sind beispielsweise:

- Personalgewinnung

- Motivation zu mehr ehrenamtlichem Engagement

- Transparenz (politischer) Prozesse

- Zeitnahe Kommunikation in Krisensituationen (z. B. Bombenfund, Hochwasser, ...)

Für die Umsetzung im Rahmen der Strategie ist es wichtig, diese Punkte zu konkretisieren. „Bürger informieren“ ist ein gerne genanntes, aber wenig greifbares Ziel. Jede Information, die man veröffentlicht, informiert die Bürger schließlich in irgendeiner Form. Sinnvoll sind konkretere Formulierungen wie „Bürger zu anstehenden Wahlen motivieren“, „Image als familienfreundliche Kommune stärken“ oder „In den nächsten 12 Monaten unsere Reichweite um 20 Prozent steigern“. Im Idealfall führt schon die Formulierung der Zielsetzung zu ersten Ideen, was man in Hinwirkung auf dieses Ziel tun bzw. posten könnte. Ziele können sich sowohl auf einzelne als auch auf alle Social-Media-Auftritte der Stadt beziehen.

Diese Ziele kann man immer wieder als Kriterium heranziehen, wenn es darum geht, Themen auszuwählen und bestimmte Aspekte herauszustellen. Sie fordern 
das eigene Handeln heraus und können teilweise sogar mit Zahlen nachgewiesen werden, z. B. durch Online-Umfragen vor und nach der Familienkampagne oder durch die Statistiken der eigenen Social-Media-Kanäle. Sie haben außerdem Einfluss auf die Redaktionsplanung, denn für die Verwirklichung eines Zieles müssen Beiträge dazu in sehr regelmäßigen Abständen veröffentlicht werden.

Wen? - Zielgruppe definieren Damit ein Gespräch und letztendlich eine Beziehung zustande kommt, muss klar sein, wer am anderen Ende sitzt bzw. wen man erreichen möchte. Die häufigste Antwort auf die Frage nach der Zielgruppe lautet: „Na, ALLE?!“ Das ist grundlegend in Bezug auf die Informationen einer Stadt zwar richtig, aber nicht jede Gemeinde ist demografisch gleich aufgebaut. Und nicht jeder Bürger wird sich für alle Informationen interessieren, sondern nur für die, die aktuell, persönlich und direkt für ihn relevant sind. Hinzu kommt, dass eben nicht jeder Bürger in jedem sozialen Netzwerk vertreten ist, sondern je nach Alter und Interessen bestimmte Kanäle bevorzugt oder vermeidet.

Folgendes Bild hat sich dabei immer wieder als hilfreich erwiesen:

Stellen Sie sich vor, das Internet ist ein Marktplatz und die sozialen Medien das Straßencafé an der Ecke. Verschiedene Leute sitzen und treffen sich hier mit Freunden oder Familie, um eine angenehme Zeit zu verbringen. Es gibt viel $z u$ sehen: süße Hunde, spielende Kinder und ein Sonderangebot im Geschäft nebenan. Sie können nicht mit allen gleichzeitig sprechen, sondern möchten an einem der Tische platznehmen und mit diesen Menschen ins Gespräch kommen. Wie stellen Sie das an?

Verfassen Sie wie gewohnt eine Pressemeldung und stellen diese einfach ein, ist es, als würden Sie leise Ausdrucke auf den Tischen verteilen und wieder gehen. Wer wird die Meldung lesen? Wer wird das Gespräch mit Ihnen suchen? Wahrscheinlich niemand.

Die Personas-Methode hilft, sich zunächst ein genaues Bild von der Zielgruppe zu machen. Sie kommt ursprünglich aus der Software-Entwicklung und wird mittlerweile auch in Marketing- und Kommunikationsstrategien zielführend eingesetzt. Eine Persona ist eine fiktive Figur, die aus realen Eigenschaften der gewünschten Zielgruppen definiert wird. Sie steht beispielhaft für eine Gruppe von Bürgern. Je konkreter dabei die Beschreibung der Person, umso lebhafter wird das Bild im Kopf und umso besser kann man sich die Gesprächssituation im Café vorstellen. Genau definiert sind Personas

„(lat. Maske) (...) Nutzermodelle, die Personen einer Zielgruppe mit ihren individuellen Merkmalen charakterisieren. Sie können aufgrund ihrer umfangreichen Beschreibung helfen, sich in die Lage der Personen zu versetzen und deren Pers- 
pektive während des gesamten Entwicklungsprozesses zu vertreten. Sie werden mit einem Namen, einem Gesicht, einer Funktion, einem Werdegang und einem Privatleben versehen. Personas verfügen über Ziele und Verhaltensweisen, haben Vorlieben und Erwartungen." (Online-Marketing Praxis 2019)

Zunächst gilt es also zu klären, welche Zielgruppen man auf dem gewünschten Kanal erreichen kann und will. Dabei muss man die demografische Struktur des Netzwerks berücksichtigen. Die Silver Surfer jenseits der 60 wird man beispielsweise auf Instagram kaum erreichen. Junge Menschen zwischen 20 und 35 stellen hier die größte Nutzergruppe da. Zunächst sammelt man frei alle relevanten Zielgruppen und priorisiert sie dann unter Berücksichtigung der städtischen Themen und Zielsetzungen. Für die fünf wichtigsten sollten Persona-Steckbriefe erarbeitet werden - je detaillierter und lebendiger, desto besser. Das folgende Beispiel in Tab. 1 verdeutlicht diese Vorgehensweise.

Bereitet man nun also einen Beitrag vor, der junge Familien ansprechen soll, stellt man sich vor, wie Carina Gerstens im Straßencafé sitzt und einen Milchkaffee trinkt. Welche Aspekte des eigenen Themas sind besonders wichtig für die junge Frau? Wie kann man diese herausstellen und mit welchen Argumenten überzeugen? Welche Rückfragen könnten kommen?

Besonders effektiv wird diese Übung, wenn man einem Kollegen die Beschreibung in die Hand drückt und ihn bittet, sich in diese Rolle und Situation im Café hineinzuversetzen. Es ist ein sehr effektives, kreatives Sprungbrett, das zu einer deutlich besseren Ansprache führt.

Wo? - Netzwerke auswählen Jedes Netzwerk hat eine ihm eigene Demografie, verschiedene Themenschwerpunkte und eigene Sprach- und Umgangsformen. Die Auswahl sollte daher mit Bedacht erfolgen und nachhaltig getroffen werden. Die Anzahl der Kanäle ist abhängig von der Leistungskapazität des SocialMedia-Teams und dem Workflow im Haus.

Die sozialen Netzwerke stellen über Statistiken, Quartalsberichte und Werbetools Informationen über die vorhandenen Nutzer zur Verfügung. Diese können beim Abgleich mit der eigenen Zielgruppe und deren Mediennutzung hilfreich sein. Projekte wie „We are social“ (https://wearesocial.com) veröffentlichen in Kooperation mit Hootsuite jedes Jahr im Januar den „Digital Report“ der für verschiedene Länder detaillierte Statistiken zur Nutzung von Internet, mobilen Endgeräten, Social Media und E-Commerce liefert. Demnach sind die derzeit (Stand Januar 2019) beliebtesten Netzwerke in Deutschland YouTube, Facebook, Instagram, Pinterest und Snapchat (Himmelberg 2019). 
Tab. 1 Persona einer jungen Familienmutter, wohnhaft in Ludwigsburg, in der Nähe von Stuttgart

\begin{tabular}{|c|c|}
\hline Name & Carina Gerstens \\
\hline Alter & 34 Jahre \\
\hline Familienstand & $\begin{array}{l}\text { - verheiratet mit Torsten (37), } 2 \text { Kinder - Lena (1) und Ben (3) } \\
\text { - Wohnen mit ihrer Familie in einer 3,5-Zimmer-Wohnung (90qm) } \\
\text { und überlegen derzeit, sich eine Wohnung oder ein Haus zu kaufen }\end{array}$ \\
\hline Beruf & $\begin{array}{l}\text { Kommunikationsdesignerin, arbeitet } 3 \text { Tage/Woche fest in einer } \\
\text { Agentur und übernimmt freiberuflich noch eigene Projekte }\end{array}$ \\
\hline Einkommen & 32.000 EUR im Jahr (arbeitet seit den Kindern weniger) \\
\hline Hobbies & $\begin{array}{l}\text { Laufen, Yoga, Reisen, backt gerne - abends und am Wochenende } \\
\text { übernimmt ihr Mann das Kochen für die Familie }\end{array}$ \\
\hline Interessen/Themen & $\begin{array}{l}\text { Überlegt, das Home-Office in einen Co-Working-Space zu ver- } \\
\text { lagern oder bei einer Agentur mit einzuziehen. Immer auf der Suche } \\
\text { nach Freizeitaktivitäten mit den Kindern. Manchmal mit ihrer } \\
\text { Schwester zusammen, deren Kinder schon } 5 \text { und } 7 \text { sind. Macht sich } \\
\text { Sorgen um die Kinderbetreuung und die wenigen Plätze. Würde } \\
\text { sich gerne ehrenamtlich für ein gutes Projekt engagieren, weiß aber } \\
\text { nicht genau wo und für was. Nachhaltigkeit ist ihr wichtig. Sie ver- } \\
\text { sucht mehr und mehr Verpackungsmüll einzusparen. Hat gerade viel } \\
\text { ausgemistet und möchte zukünftiger bewusster konsumieren. Nur } \\
\text { wohin mit den alten Sachen? Kauft gerne auf dem Wochenmarkt } \\
\text { und im Biosupermarkt ein. Nutzt öffentliche Verkehrsmittel wenn } \\
\text { möglich. Kauft gerne lokal ein und unterstützt lieber die kleinen } \\
\text { Geschäfte vor Ort statt bei Amazon zu bestellen (nur im Notfall) }\end{array}$ \\
\hline Mediennutzung & $\begin{array}{l}\text { Sie hat schon lange einen Facebook-Account und ist aus beruflichen } \\
\text { Gründen auch nach wie vor recht aktiv dort. Manchmal sucht sie } \\
\text { dort nach passenden Events für die Familie, aber auch beruflich. } \\
\text { Privat verlagert sich die Kommunikation immer mehr zu WhatsApp } \\
\text { und Instagram. Auf Instagram folgt sie Freundinnen, verschiedenen } \\
\text { Elternblogs, Design-Accounts und kleineren Geschäften und Labeln } \\
\text { aus der Region. Inspiration für Wohnungseinrichtung, Urlaub mit } \\
\text { der Familie, Kinder-Aktivitäten oder auch neue Designs sucht sie } \\
\text { auf Pinterest. Beruflich ist sie auf XING und LinkedIn vertreten }\end{array}$ \\
\hline
\end{tabular}

Facebook ist nach wie vor das mit Abstand größte Netzwerk. 32 Mio. Deutsche sind hier vertreten. Das Verhältnis von Männern zu Frauen ist in etwa fünfzig-fünfzig. Den größten Anteil stellen die Altersgruppen 25-34 mit 30\%, gefolgt von den 35-44-Jährigen mit $21 \%$ (Werbeanzeigenmanager Facebook 2019). Hashtags (\#) finden hier keine sinnvolle Verwendung. Videos und Events 
zählen zu den erfolgreichsten Formaten. Zwischen ein und drei Emojis pro Beitrag sind üblich. Die Beitragsfrequenz sollte bei zwei Mal pro Woche liegen. Ende 2018 kündigte Facebook an, das Stories-Format deutlich stärker in den Fokus rücken zu wollen (Zuckerberg 2018).

Instagram ist das derzeit am schnellsten wachsende Netzwerk. Aktuell nutzen es rund 20 Mio. Deutsche. Auch hier ist das Geschlechterverhältnis ausgeglichen. Die Zielgruppe ist im Schnitt etwa 10 Jahre jünger als bei Facebook. Der Schwerpunkt liegt eher auf Teenagern und Twens (Facebook 2019). Hashtags gehören fest zu jedem Beitrag. Empfehlenswert sind vier bis sieben pro Beitrag. Sie sollten sich nicht ständig wiederholen und zum Bildmotiv passen. Königsdisziplin: Einen eigenen Hashtag etablieren. Beiträge sollten auch hier ein bis zwei Mal pro Woche erscheinen. Stories idealerweise drei bis sieben pro Tag. Mehr als 10 Emojis pro Beitrag sind nicht ungewöhnlich.

Bei Twitter dominieren die Männer mit fast $70 \%$. Gerade in Deutschland erreicht man insbesondere Journalisten sowie Technik- und medienaffine Menschen. Politiker und Parteien sowie größere Bundesbehörden sind ebenfalls zahlreich vertreten (Twitter Ads 2019). Zwischen drei und fünf Hashtags pro Tweet sind sinnvoll. Emojis sollte man wie bei Facebook eher sparsam einsetzen. Da die Halbwertszeit eines Tweets zwischen drei und sechs Stunden liegt, darf man hier gerne mehrmals täglich posten.

Pinterest ist eine visuelle Suchmaschine und wird aktuell von Frauen dominiert: Sie stellen $70 \%$ der Nutzer. Für Hochzeitsevents sowie den touristischen Bereich - insbesondere, wenn es um junge Familien als Zielgruppe geht - ein sehr attraktives Netzwerk (Pinterest 2019). Hashtags werden hier nicht eingesetzt, aber eine gute Bildbeschreibung ist wichtig. Emojis finden dagegen keine wirkliche Verwendung.

Was? - Themen auswählen Bei Auswahl der Themen spielen sowohl die eigenen Bedürfnisse eine Rolle, wie auch die Wahl des Netzwerkes und die Erwartungshaltungen und Wünsche der Bürger. Die sozialen Medien sind keine kommunikativen Einbahnstraßen, sondern sollen zum Dialog und Austausch einladen. Das entscheidende Signal an die Fans und Follower ist „Wir hören euch zu und gehen auf das ein, was euch wirklich bewegt." Die Fans und Follower können die Themen aktiv mitbestimmen.

Hierzu eine Erinnerung an das Straßencafé: Hier dürfte man sich auch nur ein einziges Mal an den Tisch setzen, wenn man einen Monolog hält, direkt im Anschluss wieder aufsteht und geht, ohne Fragen oder Entgegnungen abzuwarten. In der zwischenmenschlichen Kommunikation erscheint das völlig logisch. In den sozialen Medien scheinbar nicht. 
Bei der Auswahl der Themen ist eine Unterteilung nach Einführungsphasen und Schwierigkeitsgrad durchaus sinnvoll. Wenn man gerade erst mit dem Auftritt in einem sozialen Netzwerk startet, sich einarbeitet oder ein neues Team aufgestellt hat, gibt es Organisatorisches, dass sich erst einspielen muss. Daher bieten sich zum Einstieg eher leichtere Themen wie Tourismus, Veranstaltungen oder Stadtgeschichte an. Diese sind in der Regel positiv besetzt und können meistens mit geringem Zeitaufwand in Hinblick auf Moderation und Rückfragen umgesetzt werden.

Bei Verkehr, Bauen oder Politik kommt es dagegen häufig zu größeren und durchaus emotionalen Diskussionen. Daher ist es hilfreich, wenn man sich zu diesem Zeitpunkt schon etwas sicherer im Umgang mit dem jeweiligen Netzwerk fühlt und die Organisation im Team reibungslos läuft. Dann wiederum kann man gerade solche Themen bewusst angehen, um die Reichweite und Interaktionsrate zu erhöhen.

Die Zusammenstellung sollte dabei abwechslungsreich und interessant sein. Ein passendes Bild ist die Komposition von Musik. Einzelne Geräusche beginnen, sich zu einem Rhythmus zusammenzufügen. Es gibt laute und leise Töne, den wiederkehrenden Refrain, schnelle und langsame Stellen sowie leichte und schwierige Passagen. Wichtig ist, alles zu einem harmonischen Gesamtbild zusammenzufügen.

Für Interaktionsraten und Reichweite dürfen es auch einmal leichtere und unterhaltsamere Themen sein, z. B. der Sonnenaufgang über dem Marktplatz. Hier ist die Hemmschwelle für die Nutzer klein, sich zu äußern. Das ist der leichte Smalltalk zum Einstieg in das Gespräch am Tisch im Café. Dieser wiederum hilft, auch bei spezielleren Fachthemen mit kleiner Zielgruppe weiterhin sichtbar zu sein (als Beispiel siehe Göpping.de 2018).

Wie? - Geschichten erzählen „Kindern erzählt man Geschichten zum Einschlafen - Erwachsenen, damit sie aufwachen.“- Jorge Bucay.

Die richtige Sprache finden Schriftliche Kommunikation folgt, insbesondere in der öffentlichen Verwaltung, strengen Regeln. Diese Art der Kommunikation unterscheidet sich deutlich von der in sozialen Medien und erfordert daher eine Umstellung und etwas Übung.

Die Kommunikation in sozialen Medien folgt eher den Gegebenheiten eines persönlichen Gesprächs: Anekdoten und kleine Geschichten gepaart mit Fragen und Aufforderungen und dem Austausch mit dem Gegenüber. Auch hier hilft wieder das Bild der jeweiligen Persona im Straßencafé und bei Bedarf ein kleines Rollenspiel mit den Kollegen. Ein Praxisbeispiel aus Nürtingen verdeutlicht dies: 
Die neuen Ausbildungsplätze sollen beworben werden. Der Verantwortliche ist selbst noch unter 30 und somit recht nah an der Zielgruppe. Seine Kollegen beide über 50 - versetzen sich in die Rolle vom zwei Teenagern im Straßencafé. Er liest seinen vorbereiteten Facebook-Post vor und versucht mit Flyern in der Hand ins Gespräch zu kommen: „Bei uns gibt es tolle und abwechslungsreiche Ausbildungsstellen. Habt ihr nicht Lust, euch bei uns zu bewerben?" Die beiden Kollegen schauen nur kurz auf und wenden sich gelangweilt ab. Er startet nach kurzem Zögern einen neuen Versuch: „Darf ich euch mal etwas fragen? Was macht für euch eine gute Ausbildungsstelle aus?" Die beiden Kollegen beginnen lebhaft zu erzählen: Keine Überstunden, damit genug Zeit für die Fußballmannschaft bleibt. Keine Langeweile im Büro, sondern lieber draußen unterwegs sein. Daraus entstand letztendlich die Idee, die Stellen über die Betriebssportmannschaft zu bewerben und auch weniger klassische Berufe wie Forstwirt, Eventmanager oder Koch vorzustellen.

Wichtig ist, bei der Formulierung auf Augenhöhe mit der Zielgruppe zu sein und ein möglichst gutes Gespür dafür zu entwickeln, was diese wirklich bewegt. Der Schreibstil sollte sich dabei stärker an der gesprochenen Sprache orientieren.

Psychoanalyse für Einsteiger - Was will die Zielgruppe wirklich? Gute Beiträge sprechen dabei keine oberflächlichen Informationen an, sondern die tiefer liegenden Beweg- und Motivationsgründe der Zielgruppen. Es lohnt sich, tiefer einzusteigen, um die Ansprache zu optimieren, wie das Beispiel in Tab. 2 zeigt.

In diesem Fall wäre es also sinnvoller, nicht nur auf das Thema ,Verfügbarkeit von Kindergartenplätzen“ einzugehen, sondern den Eltern zu vermitteln, wie gut es den Kindern dort geht, wie sie gefördert werden und, dass auch die Stadt bemüht ist, den Kindern die bestmögliche Betreuung zukommen zu lassen.

Die eigene Rolle - Held oder Mentor? In jeder guten Geschichte gibt es eine Hauptfigur - den Helden. Er wird durch äußere Umstände dazu gebracht, sich auf

Tab. 2 Beispiel für Beweg- und Motivationsgründe der Zielgruppe

\begin{tabular}{l|l}
\hline Grundlegendes Problem & Ich brauche einen Kindergartenplatz für mein Kind \\
\hline Innere Fragestellungen & $\begin{array}{l}\text { Wird mein Kind liebevoll betreut? Sind die Erzieher sympa- } \\
\text { thisch und haben ähnliche Werte wie ich? Fühlt es sich mit den } \\
\text { anderen Kindern wohl? Wird es gefördert? }\end{array}$ \\
\hline Philosophisch & Ich möchte das Beste für mein Kind! \\
\hline Hürde/Problem & Es gibt wenige Plätze und ich bin darauf angewiesen \\
\hline
\end{tabular}


eine Reise zu begeben, die entweder im Glück oder im Unglück endet. Nun ist die Frage: Wer übernimmt in der Geschichte die Rolle des Helden und wer die des Mentors? Wer ist Yoda und wer Luke Skywalker?

Im Fokus der Geschichte sollte immer die Zielgruppe stehen. Die Kommune übernimmt also die Rolle des Mentors. Sie ist empathisch für die Situation der Zielgruppe, hat aber das notwendige Wissen und die Autorität mit Wissen oder einem konkreten Plan weiterhelfen zu können, damit die ,Heldenreise“ ein gutes Ende nimmt.

Emotionen sind das Salz in der Suppe Gute Geschichten und Dialoge leben von Emotionen. Emotionen dienen psychologisch dazu, Menschen zu Handlungen zu aktivieren und diese wiederum auf das Erreichen eines bestimmten Zieles auszurichten. Mit der Einführung der ,Reactions“ im Jahr 2014 hat Facebook der Basispalette der menschlichen Empfindungen Rechnung getragen. Statt einem einfachen ,Gefällt mir' kann man nun auch Liebe, Überraschung, Wut oder Trauer mit einem Klick ausdrücken.

Eine Studie von FanpageKarma (2016) hat dabei gezeigt, dass diese emotionalen Reaktionen auch Auswirkungen auf die Reichweite der Beiträge haben und verstärkt zu Interaktionen animieren. Die positiven Emotionen Liebe, Lachen und Überraschung erhöhen die Reichweite im Schnitt um das Dreifache, Trauer und Wut immerhin noch um das Zweifache im Vergleich mit einem einfachen ,Gefällt mir‘.

Möchte man als Seitenbetreiber organisch die Reichweite erhöhen, kann also das Erzeugen einer passenden Emotion hilfreich sein. Eine rein sachliche Information wird nur in seltenen Fällen eine gefühlvolle Reaktion hervorrufen. In die Formulierung eines guten Beitrags fließen gezielt die passenden Gemütsbewegungen mit ein, die man beim Leser auslösen möchte. Man lässt die Fans und Follower an den eigenen Gefühlen teilhaben und schafft so sympathische Momente, mit denen sich die Community identifizieren kann.

Eine gute Gesamtgeschichte besteht jedoch nicht nur aus schönen Erlebnissen. Sie zeigt auch Schwierigkeiten, Hindernisse, Tränen und Schweiß. Erst durch die ganze Palette der menschlichen Emotionen wird das Bild facettenreich, nahbar und echt. An diesem Punkt gibt es häufig die größten Widerstände: „Wir können doch keine Fehler oder Schwächen nach außen kommunizieren! Was sollen denn die Bürger denken?" Natürlich gilt es hier gut abzuwägen. Aber es ist die Chance zu zeigen, wie es um Fehlerkultur und Problemlösungskompetenzen in der Verwaltung bestellt ist.

„Die Sprache der Nutzer zu sprechen ist das A und O“ (Dejan Birk-Mrkaja, Göppingen) In Hinblick auf die Sprache hilft es, sich inspirierende ,Vorbilder" 
außerhalb der öffentlichen Verwaltung zu suchen. Social-Media-Verantwortliche müssen sich mit der Kommunikation in den ausgewählten Netzwerken auseinandersetzen. Jedes von ihnen hat Besonderheiten und eigene Regeln, die man verstehen sollte - sonst wirkt das städtische Profil schnell wie ein Fremdkörper. Dazu gehören neben Wortwahl und Ansprache (Du vs. Ihr vs. Sie) auch die Verwendung von Hashtags, Emojis und GIFs. Experimente sind durchaus erlaubt. Grundsätzlich gilt: die ersten 150 Zeichen sind in fast allen Netzwerken gleichermaßen entscheidend. Diese überfliegen die Nutzer, um dann zu entscheiden, ob sie weiterlesen und interagieren oder weiterscrollen. Humor und Selbstbewusstsein geben dem Ganzen die richtige Würze.

\section{$5 \quad$ Zusammenfassung}

Die Basis für einen erfolgreichen Social-Media-Auftritt ist ein gut aufgestelltes und organisiertes Team, dass sowohl mit Wissen als auch mit den notwendigen Werkzeugen ausgestattet ist, um effizient arbeiten zu können.

Inhaltlich sollte jeder Beitrag die folgenden Aspekte beantworten können:

- Auf welches (langfristige) Ziel zahlt der Beitrag ein?

- Welche Zielgruppe wird angesprochen?

- Was bewegt die Zielgruppe (innerlich/philosophisch)?

- Welches Netzwerk ist geeignet?

- Wie lässt sich der Inhalt am besten vermitteln? (Sprache, Medien, Emotionen, Hashtags)

- Welche Emotion soll ausgelöst werden?

- Welcher Zeitpunkt eignet sich dafür?

Wenn dann Bildsprache und Wortwahl sowie der Einsatz von Hashtags und Emojis passend zum Netzwerk gewählt werden, sollte dem Erfolg nichts mehr im Wege stehen.

\section{Literatur}

Facebook. (2019). Quaterly earnings. https://investor.fb.com/financials/default.aspx. Zugegriffen: 19. März 2019.

FanpageKarma. (2016). [Infografik]. Facebook Reactions. Mehr Reichweite und Interaktion. https://blog.fanpagekarma.com/2016/04/05/infografik-facebook-reactions-mehrreichtweite-und-interaktion/?lang=de. Zugegriffen: 20. März 2019. 
Göpping.de. (2018). Babyvornamen. Facebook-Post. https://www.facebook.com/Stadt. Goeppingen/photos/a.333740466650969/2628815633810096/?type=3\&theater. Zugegriffen: 20. März 2019.

Himmelberg, C. (2019). Digital 2019. Deutschlands digitales Verhalten. https://wearesocial.com/de/blog/2019/01/digital-2019-deutschlands-digitales-verhalten. Zugegriffen: 20. März 2019.

Nürtinger Schlemmer Sommer. (2018). Facebook-Video. https://www.facebook.com/Nuertingen.de/videos/533234263767795/. Zugegriffen: 8. Mai 2019.

Online-Marketing Praxis. (2019). Definition Personas. https://www.onlinemarketing-praxis.de/glossar/personas. Zugegriffen: 7. Mai 2019.

Pinterest. (2019). Nutzerstruktur. https://analytics.pinterest.com/audience-insights/?tab=pinterest\&audience_1=pinterest___impression_plus_engagement. Zugegriffen: 20. März 2019.

Roth, P. (2017). Der Facebook Newsfeed Algorithmus. Die Faktoren für die organische Reichweite im Überblick. https://allfacebook.de/pages/facebook-newsfeed-algorithmusfaktoren. Zugegriffen: 19. März 2019.

Twitter Ads. (2019). Werben auf Twitter. https://ads.twitter.com/login. Zugegriffen: 19. März 2019.

Werbeanzeigenmanager Facebook. (2019). Werbeanzeigen erstellen. https://www.facebook. com/business/help/182715205255643. Zugegriffen: 20. März 2019.

Zuckerberg, M. (2018). Mark Zuckerberg auf Facebook. https://www.facebook.com/zuck/ posts/10105349847863791. Zugegriffen: 7. Mai 2019.

Open Access Dieses Kapitel wird unter der Creative Commons Namensnennung 4.0 International Lizenz (http://creativecommons.org/licenses/by/4.0/deed.de) veröffentlicht, welche die Nutzung, Vervielfältigung, Bearbeitung, Verbreitung und Wiedergabe in jeglichem Medium und Format erlaubt, sofern Sie den/die ursprünglichen Autor(en) und die Quelle ordnungsgemäß nennen, einen Link zur Creative Commons Lizenz beifügen und angeben, ob Änderungen vorgenommen wurden.

Die in diesem Kapitel enthaltenen Bilder und sonstiges Drittmaterial unterliegen ebenfalls der genannten Creative Commons Lizenz, sofern sich aus der Abbildungslegende nichts anderes ergibt. Sofern das betreffende Material nicht unter der genannten Creative Commons Lizenz steht und die betreffende Handlung nicht nach gesetzlichen Vorschriften erlaubt ist, ist für die oben aufgeführten Weiterverwendungen des Materials die Einwilligung des jeweiligen Rechteinhabers einzuholen.

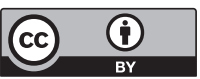

